\title{
THE CENTRIPETAL PATHWAY FROM THE BLADDER AND URETHRA WITHIN THE SPINAL CORD
}

\author{
BY
}

\author{
P. W. NATHAN and MARION C. SMITH
}

From the Neurological Research Unit of the Medical Research Council, The National Hospital, Queen Square, London

While clinical and experimental facts about lesions of the spinal cord and the functioning of the bladder have been collected for more than 50 years, knowledge remains inadequate concerning the afferent pathway from the bladder within the central nervous system of man. In 1906, von Frankl-Hochwart and Zuckerkandl wrote in their monograph on neurological disorders of the bladder: "Sehr gering ist noch unser Wissen über die spinalen Leitungsbahnen; am Menschen ist man nie über unbeweisbare Hypothesen hinausgekommen". They also stated specifically that nothing was known about the ascending tracts concerned with conduction from the bladder. In 1919, Müller still found it necessary to write : "Über die Bahnen im Rückenmark, durch welche die Empfindungen von den Blasenkontraktionen zentralwärts geleitet werden, und über die Stelle im Gehirn, wohin diese projeziert werden, sind wir noch völlig im Unklaren". In 1939, Bing and Haymaker wrote: "The pathways which the afferent fibres follow to reach the conscious level are not known".

Barrington investigated the problems of localization of the central nervous pathways by means of experimental lesions in animals. In 1925, he reported the results that he had obtained by making lesions in the midbrain of the cat ; he found that "destruction of the midbrain from the ventral half of the side of the posterior end of the aqueduct outwards to just beyond the mesencephalic root of the fifth nerve is followed when the lesion is bilateral by a permanent loss of consciousness of wanting to micturate or defaecate". In 1933, he reported further work, showing the position of the afferent pathway in the spinal cord of the cat. He concluded that the whole or a large part of the centripetal pathway must lie in a superficial area in the posterior part of the lateral columns, not reaching as far posteriorly as to abut against the root entry zones. This region is shown in Fig. 1

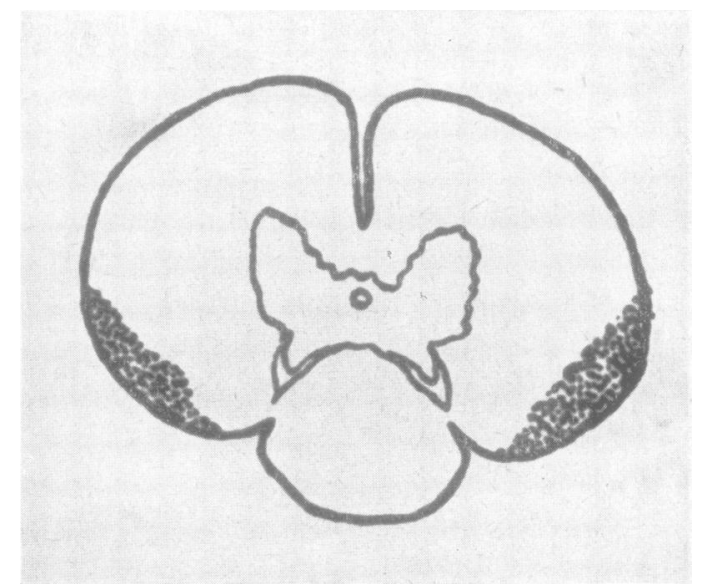

Fig. 1.-Diagram of transverse section of spinal cord of cat. The area within which the centripetal bladder pathway lies is shown by stippling.

(a reproduction of Fig. 4 of Barrington's paper). Among the many clinical papers which are concerned with the effects of lesions of the central nervous system on bladder function, we know of only one which considers the afferent tract, that by McMichael (1945), who reported the case of a patient who had lost sensation of bladder fullness. Sections of the spinal cord showed an area of degeneration anterior to the posterior horns in the lateral columns. This area could not be traced cranial to the thoracic region.

It is the purpose of this paper to present evidence concerning the location within the human spinal cord of the centripetal pathways (1) from the bladder, conveying impulses subserving $(a)$ the desire to micturate, (b) pain ; and (2) from the urethra, conveying impulses subserving $(a)$ touch, (b) pain, (c) temperature, and (d) proprioception from the muscles working in association with the urethra. 


\section{Material}

The material consists of 27 patients with lesions of one or both antero-lateral columns of the spinal cord, and of three control patients, in whom these regions were intact. Of the 27 patients, 24 had the operation of anterolateral cordotomy performed for pain associated with cancer; one had this operation performed for the pain of tuberculous cystitis; and two patients had arterial thromboses, one of the anterior spinal artery, and one of a posterior inferior cerebellar artery. The patients of the control group suffered from amyotrophic lateral sclerosis, subacute combined degeneration of the cord, and from Friedreich's ataxia. Histological preparations have been made of 18 of the first group, and of all of the control groups.

The operations of spino-thalamic cordotomy were carried out for the relief of pain. The area of cord transected, although planned to give the maximum relief of pain, was varied to some extent from patient to patient. Seventeen of the patients were under the care of Mr. McKissock or in the wards of the Research Unit ; they were fully investigated on several occasions before and after operation. Through the kindness of other neurosurgeons, other patients have been made available to us; these cases have been investigated only after operation. As the surgical interference has been undertaken for the relief of pain associated with cancer, the survival period has been relatively short; therefore it has been possible to determine histologically the extent of the incision in the cord and the resulting ascending and descending degeneration.

\section{Investigation}

Clinical.-The investigation falls into two sections, clinical and histological.

Bladder Sensation.-The main clinical evidence is based on day to day observation of the patient. Special investigations often added little to what was obvious on clinical observation. For example, if a patient is lying in bed with the bladder distended so that it can be felt as a dome between the pubic crest and the umbilicus, and the patient is quite unaware of this state of extreme distension, it can be concluded without further investigations that the patient has lost the sensation of needing to micturate and that of pain from over-distension of the bladder. Wherever possible, however, conclusions drawn from these observations were confirmed by observing what occurred under the controlled conditions of cystometrography.

Immediately after the operation of spino-thalamic cordotomy, which, when performed bilaterally, caused retention of urine, the bladder was allowed to become distended in order to find out what sensations were experienced; catheterization was then performed to relieve this distension. Cystometrography was carried out before and after operation. It was possible in this way to record the conditions of pressure and fluid content which gave rise to any form of vesical sensation, and to compare records made before and after the operation. The patients were asked to report any sensations they experienced. The evidence thus depends entirely on the subjective experience of the patient.

The method of cystometrography employed was designed to imitate as closely as possible the normal filling of the bladder. After the bladder had been emptied by catheterization, fluid-a warmed solution of proflavine sulphate, 1 in 10,000 in normal saline-was allowed to flow through a catheter into the bladder, at a rate of one to two drops a second. The pressure at which the fluid entered the bladder was the same as that which the bladder exerted on its contents.

A drawing of the apparatus is shown in Fig. 2. The proflavine solution, stored in a graduated reservoir, passed through a drip-bulb, the rate of flow being adjusted by a screw-clip. The drip-tube was connected by three channels; first to the catheter, which had previously been fixed in the bladder, secondly to a manometer, consisting of an upright glass tube, open to the air, and thirdly to a hollow C-spring, attached to the end of which was an ink-writer. The pressure in the bladder at any given moment could be read from the height of the fluid in the manometer. While the record was being made, the patient was invited to say what sensations, if any, were experienced. It will be noted that the amount introduced into the bladder from the reservoir was not necessarily the same as the fluid contents of the bladder, because the kidneys were secreting urine throughout the investigation. The total fluid content was obtainable only at the end of the investigation, when the total contents withdrawn by catheter were measured.

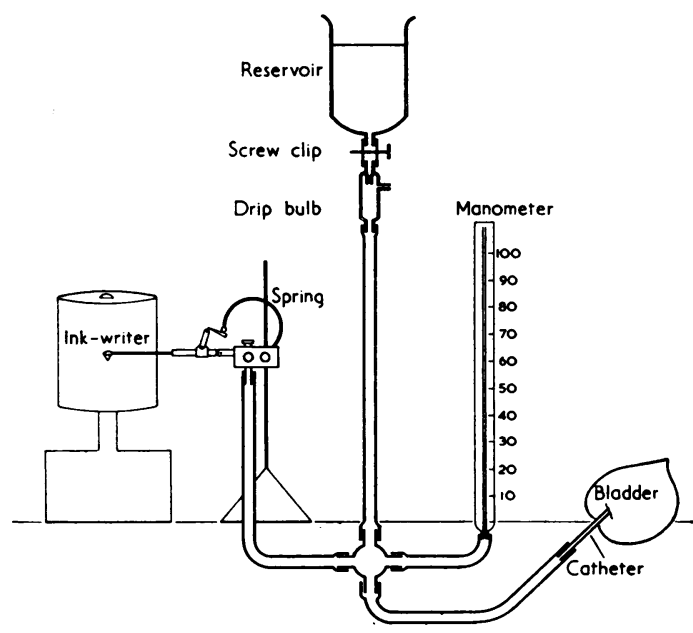

FIG. 2

Pain sensibility in the bladder was investigated in various ways. During cystoscopy, which was performed before and after operation, attempts were made to induce pain by indenting and stretching the neck of the bladder, the trigone, the bladder walls, and the ureteric orifices. The loss of painful sensation, when the bladder 
was allowed to become distended after the bilateral operation, was taken to indicate that the pathway subserving the pain of bladder distension had been divided. In some patients, the spino-thalamic cordotomy had been performed for a painful condition of the bladder ; if the operation relieved this pain, then the pathway subserving pain in the bladder must have been divided. In other patients, who have had the operation of bilateral cordotomy, a condition of the bladder has been found post-mortem that is known to be painful ; such are inflammation of the viscus and ulceration. As there were no complaints of pain during life, it was concluded that the operation cut the tracts conveying impulses interpreted as pain. It is therefore possible to determine by analysis the centripetal pathway subserving pain caused by a variety of bladder conditions.

Urethral Sensation.-From the normal urethra impulses are transmitted subserving sensations of touch, of warmth and cold, and of pain. In addition, one of the main sources of sensation associated with micturition is proprioception from the muscles that act in association with opening and closing of the sphincter. The sensations of pain, touch, and of forceful opening of the sphincter by a catheter or cystoscope were noted before and after operation. The patients were also asked to observe what they felt when they passed urine voluntarily or involuntarily.

As it is not yet definitely known whether the bladder mucosa is sensitive to thermal stimuli, and as the urethral mucosa definitely is sensitive to such stimuli, no attempt was made here to differentiate the site of origin of the thermal sensations experienced. Proflavine solution at various temperatures was introduced into the bladder through a urethral catheter before and after operation. The patient was asked to comment on the sensations experienced.

Histological.-Fixation of the spinal cord was initiated as soon after death as possible. In the majority of cases the cord was fixed in situ; $20 \%$ formol saline was injected into the cisterna magna, while cerebrospinal fluid was drained by lumbar puncture ; after a few hours the brain and cord were removed, and the necropsy completed. Fixation of the brain and cord in $20 \%$ formol saline was continued for 10 to 18 days, and the cord was then divided into segments. To demonstrate degenerated pathways, the techniques used were the Swank Davenport modification of the Marchi method, the Kulchitsky modification of the Weigert Pal method, and Gros' silver method. The blocks were embedded in celloidin.

Sections were taken at several levels of each segment of the cord, and in areas of special interest, serial sections were prepared. In the majority of cases the modified Marchi method was found to give satisfactory results, even when the interval between operation and death was about a year (Smith, 1951). Silver and Weigert Pal preparations were used to confirm the findings shown by Marchi staining. It was considered necessary to use the silver method in order to show destruction of axis cylinders, for the myelin stains may not demonstrate the absence of small, poorly myelinated fibres.

\section{Clinical Evidence}

In normal subjects, the vesical pressure at which sensations are experienced varies considerably from time to time, and from subject to subject. Also, the sensations experienced are related to the rate of filling, and to the rate of change of pressure. However, in all normal subjects, when the bladder contains a certain quantity of fluid, a stage is reached when there is a definite desire to micturate. In the present investigation, 20 healthy men and women were examined by the method of cystometrography described. An average of the figures from these subjects showed that there was a definite, insistent desire to micturate when the bladder contained $340 \mathrm{ml}$. of fluid at a pressure of $14 \mathrm{~cm}$. of water.

The clinical evidence is presented in a condensed form as Table I. Those cases marked with an asterisk are mentioned in further detail.

Case 9.- Immediately following the second stage of a bilateral sympathectomy this patient developed a thrombosis of the anterior spinal artery. This caused complete analgesia throughout the right side below the second lumbar dermatome and throughout the left side below the third lumbar dermatome. There was retention of urine ; the patient had no normal desire to micturate, and no pain with the retention. Eventually she got a feeling whenever her bladder was distended, which she described as follows : "My tummy feels uncomfortable ; it is a feeling of wind really ; I cannot tell which it is ; it is not the normal feeling of having to empty the bladder, only an uncomfortable feeling in the tummy ". She could feel a catheter being passed, but it was entirely painless. Often, when bed-pans were being taken round the ward, she would get a normal feeling of needing to micturate, but it lasted only a minute or two, and would be unrelated to the state of distension of her own bladder.

Case 10. - This case showed much degeneration of all afferent pathways ; it is included in this series, as it is adequate for supplementary evidence, and because this patient, a man of 21, gave such good descriptions of his sensations. Following the operation of bilateral cordotomy, pain sense was diminished below the eleventh thoracic dermatome on the right, and absent throughout the left below the tenth thoracic dermatome. There was retention of urine, but the patient was unaware of this. He soon found, however, that he could tell when his bladder was full, as he got a sensation which he described as follows : "When my belly feels very full, as though I have eaten a lot, I know I have to empty my bladder". The normal sensation that his bladder needed emptying had gone. This patient later explained that the feeling of abdominal fullness which he got when his bladder was full, seemed to him to be unrelated to his bladder; it seemed to occur vaguely and generally. within his abdomen; sometimes this sensation also occurred when his abdomen was full of wind or his rectum full of faeces ; in itself the sensation was non- 
specific ; he learnt by experience that it usually meant that his bladder needed emptying. When he was being catheterized, he felt nothing until the tip of the catheter passed the membranous urethra, when he explained that he had merely the feeling of the presence of something in that region; it was a painless sensation. Before operation catheterization had been painful, and had left a soreness afterwards.

When micturition became automatic he would get, just before it began, a tingling and itching in the penis. This sensation could also be induced by giving $0.25 \mathrm{mg}$. of carbaminoyl choline (carbachol), which would cause urine to be voided.

Case 18. - This patient before bilateral cordotomy had such terrible pain every time he micturated that often he had to be given " trilene" by inhalation. His bladder was shrunken, and he had to micturate every five or 10 minutes. A bilateral antero-lateral cordotomy was performed by Mr. O'Connell under local anaesthesia. By the time the cord was exposed, the patient had a most urgent desire to micturate and much pain in the bladder. When the cord had been bilaterally incised, the pain and the desire to micturate were simultaneously relieved. After the operation, which caused analgesia below the fourth thoracic dermatome, the patient eventually had to empty his bladder only once every hour. He was able to do this while carrying on a conversation, a state of affairs quite unlike that which had prevailed before the cordotomy.

Case 19.-Following bilateral cordotomy there was complete analgesia throughout the body below the fourth thoracic dermatome. On the evening of the day of operation, the patient was catheterized on account of retention of urine: he noted that he felt the passage of the catheter, although it was painless, whereas before operation it had been so painful that it had almost to be given up. On the next day, he was found with his bladder full and distended, so that it could be felt above the pubic crest. He was moaning on account of the pain of it. He said that the bladder felt full, although the sensation was a new one, and not the normal feeling of urgently needing to micturate ; it differed in that it was not felt in the penis or perineum, but was situated only in the lower abdomen, above the pubic crest. When his distended bladder was pressed on through the abdominal wall, the pain was increased. It was found that he got no pain on having his testicles squeezed, his pubic hair vigorously pulled, and that pinprick on his penis and scrotum was felt only as touch. This lower abdominal pain was associated with bilateral frontal headache; both pain and headache went when the bladder was emptied. He gradually developed more sensation in the urethra. When the catheter was passed, he got a tickle in the penis ; sometimes he got a pricking or irritation at the meatus; as it passed along the membranous urethra he got a desire to micturate, and this feeling always became intense when the tip of the catheter reached the membranous part. Then he found that whenever micturition was about to begin, he would get the same pricking feeling in the penis; he also got it when he strained to micturate, and also if too big a catheter reached the posterior urethra. He had no normal feeling that his bladder was full and no normal desire to micturate ; when it was very full, he sometimes got a pricking sensation above the pubic crest, which would go away as urine was let out. Four weeks after the operation, he again had some pain in his bladder and in the tip of the penis ; the bladder contained $640 \mathrm{ml}$. of urine. He stated that this pain was the normal sensation one has when one needs urgently to micturate.

Case 20.-Following unilateral cordotomy there was analgesia throughout the right side of the body below the fifth thoracic dermatome. There was for a few days after the operation " not a pain, well, yes-a pain, but only slight" immediately above the pubis and just to the left of the midline, when he needed to micturate. He then found that he had the feeling of needing to micturate only on the left side, the side of the body that was normally innervated. Later, he felt it mainly on the left, and eventually he noticed no laterality in the sensation, having a normal sensation underlying the desire to micturate.

Case 23.-Following deep $x$-ray treatment, the patient got severe pain in the left thigh and knee; he also got a strange feeling of warmth running down the left thigh when he micturated; this was accompanied by frequency of micturition. During cystoscopy, the following observations were made. As the cystoscope reached the posterior urethra, he had a desire to micturate; he got the same desire when this part of the urethra was stretched. The bladder was filled at a pressure of $50 \mathrm{~cm}$., and when it contained $600 \mathrm{ml}$. he got an urgent need to micturate. Some of the fluid was released; then rubbing of the trigone with the cystoscope again brought on the desire to micturate. A ureteric catheter was then put into the right ureter; this gave him the feeling of a pricking on the right side of the penis ; as the ureteric orifice was stretched, the pricking feeling became a continuous pain; as the catheter was pushed up the ureter, it felt as though " you are pushing in a tender place on the right ". On the left half of the bladder, there were many telangiectases, doubtless due to radiotherapy ; the slightest touch over these with the tip of the ureteric catheter caused severe pain, felt either on the left or just to the left of the midline or centrally. The left side of the bladder was so tender, that search for the ureteric orifice had to be given up.

Following unilateral cordotomy there was total analgesia throughout the left side below the twelfth thoracic dermatome. Cystoscopy was repeated three weeks after operation. The bladder was filled at a pressure of $50 \mathrm{~cm}$.; when $700 \mathrm{ml}$. had been put in, the patient got an urgent desire to micturate, only on the right side. The ureteric catheter was pressed into the lateral walls ; nothing was felt on the left, a slight pain was felt on the right. The telangiectases, so sensitive before the operation, were rubbed vigorously (they occurred only on the left side of the bladder); nothing was felt. Both ureteric orifices were then stretched as much as possible by means of the ureteric catheter ; nothing was felt on the left ; on the right, penetration caused a slight feeling of pressure, stretching caused a 


\section{TABLE}

OBSERVATIONS ON BLADDFR AND URETHRAL SENSATION MADE ON 27 PATIENTS WIOPH

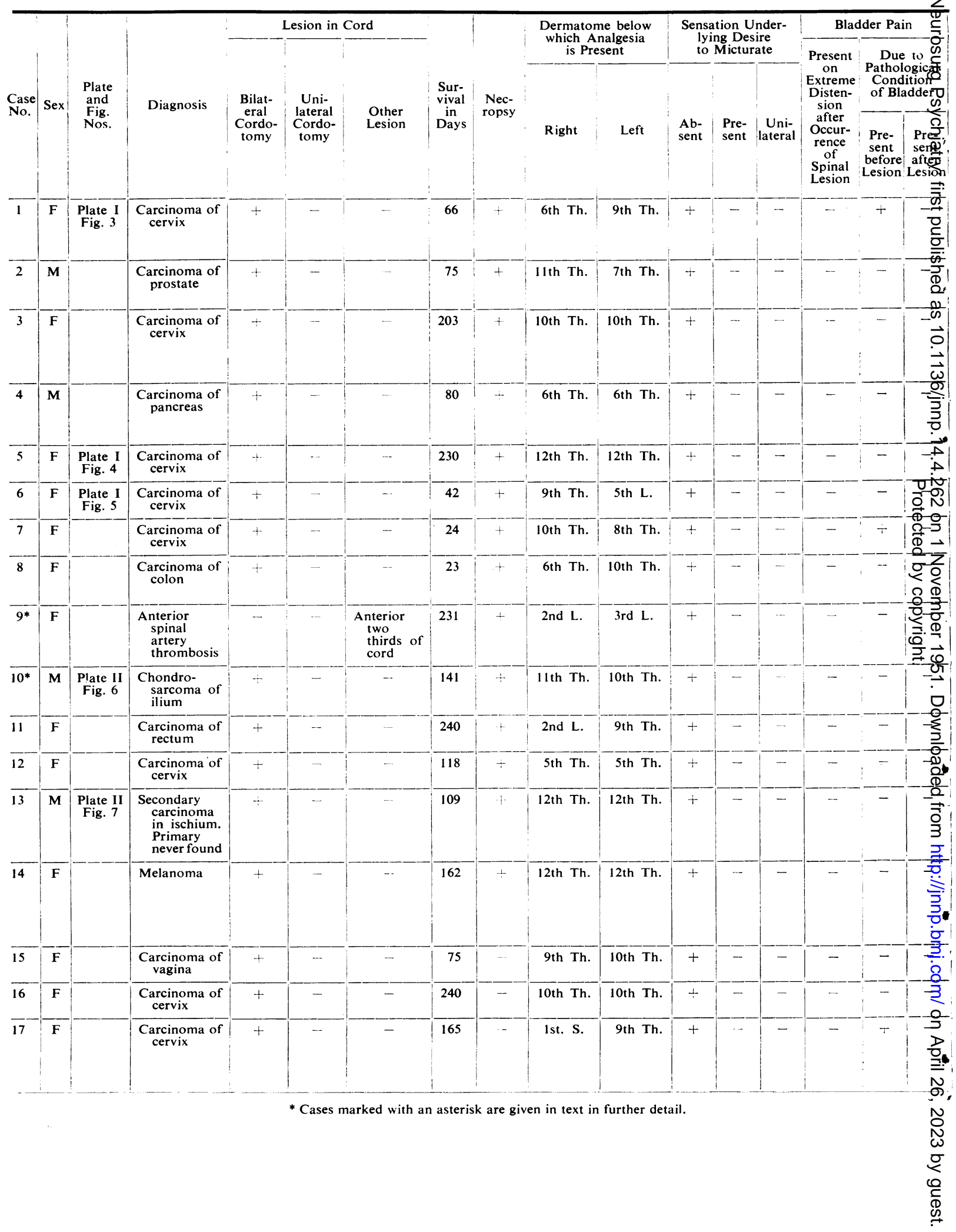




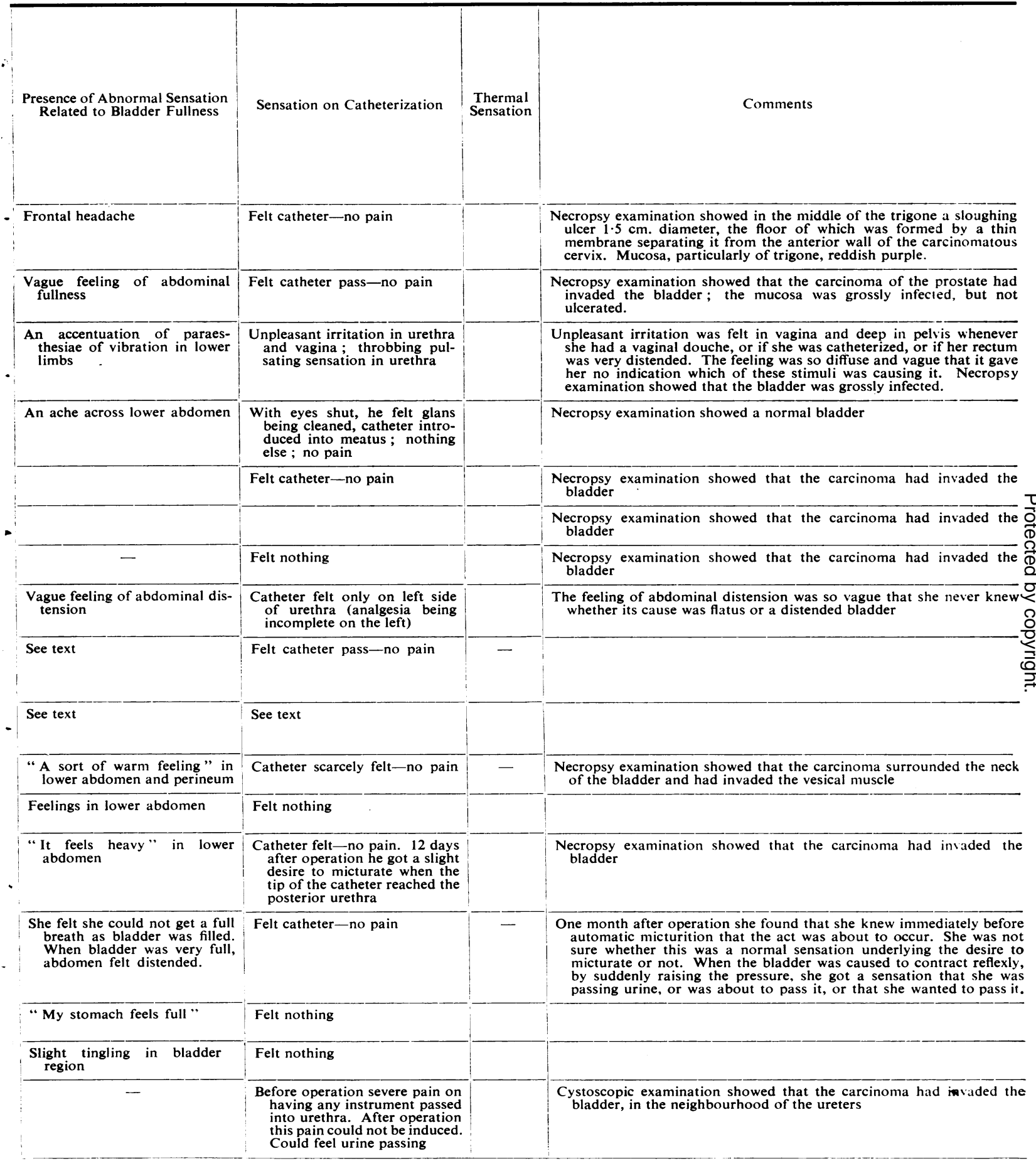


TABLE I-Z

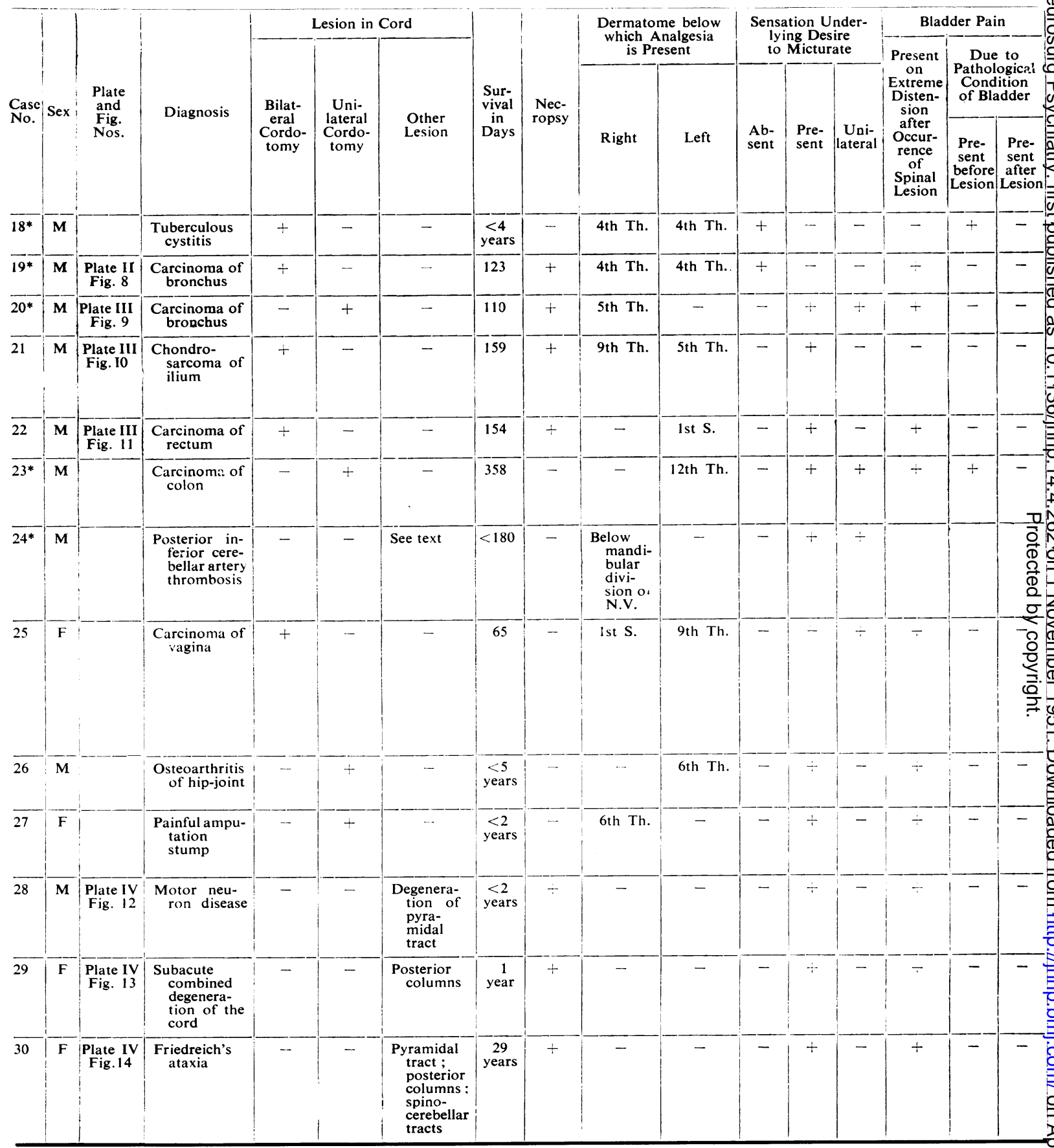

* Cases marked with an asterisk are given in text in further detail. 


\section{(Continued)}

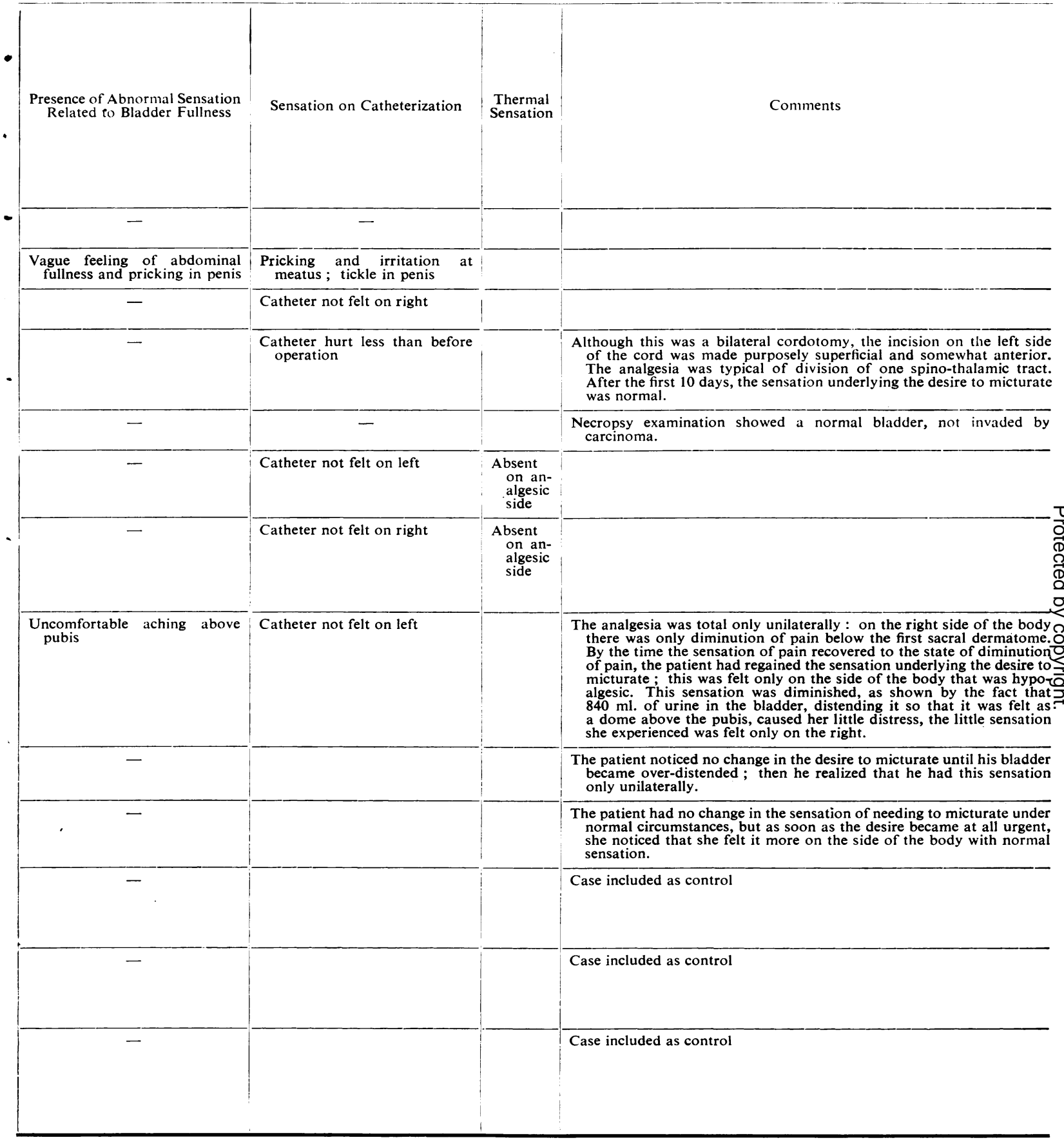


pain felt on the right. Pressure with the cystoscope on the trigone caused a severe urge to micturate, felt only on the right; pressure on the bladder neck in the midline caused a feeling of pressure on the right side of the pubic crest. The ureteric catheter was then pushed hard in the midline into the wall of the bladder at the junction of the posterior wall and the dome ; this caused no sensation on the left, and a moderate pain just to the right of the midline of the pubic crest. Strong pressure was then applied with the cystoscope on the left side of the bladder neck; this caused no sensation on the left, but a sharp pain on the right, of the kind that accompanies an urgent need to micturate. It will be realized that actually pressure in this region cannot be made unilaterally, so that pressure on the left must have pulled on and distorted the right half of the bladder neck and posterior urethra. Two days later cystometrography was carried out. The catheter was felt only on the right. All the sensation that he needed to micturate, and finally, the urgent desire to micturate, were felt only on the right. When it was pointed out to the patient that it was difficult to imagine such a sensation, he insisted that it was so, saying that he felt the full state of his bladder on the right side of his penis, on the right side of his perineum, and generally in the pubic region on the right. When $675 \mathrm{ml}$. of fluid had been run in, the rate of flow was increased. The patient, who was an excellent observer, said that he could feel his bladder getting fuller, almost drop by drop, but only on the right.

The subjective symptoms related to micturition referred to above ceased after the operation.

Case 24.-This patient was seen a few months after the onset of a thrombosis of the left posterior inferior cerebellar artery. As he is still alive, no histological evidence about the extent of the lesion has been obtained. Although there may be minor differences in the region of the medulla involved by thrombosis of this artery, all cases show involvement of the spino-thalamic tract. In this patient pain and thermal sensation throughout the right side of the body below the third division of the fifth cranial nerve were absent. In reply to questioning, the patient stated that he had noticed no change in any way in micturition, and that he had the usual sensation underlying the desire to micturate. However, when cystometrography was carried out, he was asked to pay attention to his sensations. He then found that he felt the sensation underlying the desire to micturate only and entirely on the left; it was located mainly in the left side of the penis. From that time onwards he realized that he experienced this sensation only on the left side, and he found that when he passed urine he felt it passing only on the left. When cystoscopy was performed later, he felt the instrument being passed only on the left. The posterior urethra was then stretched by elevation of the bridge on the instrument : on the right this caused no sensation ; on the left, it caused a feeling of pricking in the left of the penis. Catheters were then pushed into the ureteric orifices, and the orifices and lower ends of the ureters were stretched; something was felt on the left, nothing on the right. A ureteric catheter was pushed into the wall of the bladder on the right side, so as to deform the whole viscus ; this caused some sensation, located to the right. It should be pointed out, however, that by this time, there was some recovery of pain conduction, so that severe stimulation of the skin on the right with a pin caused a slight sensation of pain.

From Table I, it will be seen that many of those patients who had lost the normal sensation underlying the desire to micturate experienced other sensations related to bladder filling and emptying. These sensations may now be considered, under the following headings : (a) abnormal sensations related to bladder fullness; (b) sensations at a distance ; (c) urethral sensations and the sensation underlying the desire to micturate.

\section{Comments on Clinical Evidence}

(a) Abnormal Sensations Related to Bladder Fullness.-Repeated observations were made by the patients who had lost the normal sensation underlying the desire to micturate on a certain new sensation in the lower abdomen-which might be called a substitute sensation-by means of which they were made aware of the state of tension in their bladders. It consisted of a vague feeling of fullness, poorly localized, usually described as occurring " down below ", or " in the stomach" (" stomach" being used by many people in this country as a euphemism for belly or abdomen). Further comment on this sensation will be made in the discussion; it suffices to state here that it was never confused with the normal sensation by any patient.

(b) Sensations at a Distance.-Sensations at a distance are those sensations, such as frontal headache, the feeling one is about to shiver, or that one cannot take a deep breath, which have been observed and investigated by Guttmann and Whitteridge (1947) in cases of complete division of the cord. As has been shown, such sensations occurred also in these cases, where the cord was never divided, and where only the afferent pathway from the bladder was divided.

(c) Urethral Sensations and Sensation Underlying Desire to Micturate.-It is known that in the normal subject the sensation of having a full bladder that needs emptying is related to a rise in tension of the bladder wall. But in some normal subjects various forms of stimulation of the urethra cause a desire to micturate. Such people experience this desire when a catheter or cystoscope is passed along the urethra, and particularly when its tip stretches or deforms the membranous and prostatic parts. In such circumstances, however, there is no rise in bladder tension. Yet these subjects may find that this sensation is the same as that which 
they experience when their bladders are full and under tension, or they may find that it consists of only the urethral component of this sensation.

In this investigation, the sensation underlying the desire to micturate has been separated as far as possible from sensations arising in the urethra. In those patients who had bilateral lesions of the antero-lateral columns, a comparison has been made between the sensations experienced on catheterization and the sensations experienced as automatic micturition was about to start. (All these patients had lost voluntary control of micturition ; this aspect of the subject will be considered in a subsequent paper.) These sensations are listed in Table II.

It will be seen from this Table that those patients who had no urethral sensation or none but touch and stretch (the feeling of the catheter being passed or of it passing the sphincter, but without pain) had no sensation informing them of the imminence of micturition, or giving them the desire to micturate. The subject of Case 13 had the same sensation on being catheterized as she had before micturition. And the subject of Case 19 also had the same sensation on these occasions, although there were other additional sensations as the basis of a desire to micturate. It is to be noted that, with the exception of the subject of Case 16, where the sensation was probably the substitute sensation, these sensations subserving the desire to micturate all came on immediately before micturition occurred; whereas the normal sensation subserving the desire to micturate comes on many minutes before the subject chooses to micturate. It is suggested that all these patients had in fact lost the normal sensation causing the desire to micturate, but, retaining much urethral sensation, some patients interpreted the sensations associated with changes in the urethral sphincter and/or stimulation of the posterior urethra as being the normal sensation of needing to micturate.

\section{Histological Evidence}

The cases of which the clinical accounts have been given above may be divided into groups, in order that common microscopical anatomical features can be extracted.

To determine the position of the pathways, plans of each case were drawn, showing the degenerated areas at various levels of the cord. These plans from the different cases were then superimposed, and the smallest common area of degeneration at each level was determined. All the 21 cases, of which necropsy material was obtained, were used to determine the afferent pathways. As many cases show similar areas of degeneration, only
TABLE II

SENSATIONS EXPERIENCED BY PATIENTS WITH BILATERAL LESIONS OF ANTERO-LATERAL COLUMNS ON BEING CATHETERIZED AND AT THE BEGINNING OF AUTOMATIC MICTURITION

\begin{tabular}{|c|c|c|}
\hline $\begin{array}{l}\text { Case } \\
\text { No. }\end{array}$ & $\begin{array}{l}\text { Sensation on } \\
\text { Catheterization }\end{array}$ & $\begin{array}{l}\text { Sensation Occurring } \\
\text { Immediately beforc } \\
\text { Micturition }\end{array}$ \\
\hline 1 & $\begin{array}{l}\text { Catheter felt ; pain- } \\
\text { less }\end{array}$ & None \\
\hline 2 & $\begin{array}{l}\text { Catheter felt ; pain- } \\
\text { less }\end{array}$ & None \\
\hline 3 & $\begin{array}{l}\text { Irritation and throb- } \\
\text { bing }\end{array}$ & $\begin{array}{l}\text { Irritation and throbbing in } \\
\text { urethra and vagina }\end{array}$ \\
\hline 4 & $\begin{array}{l}\text { Catheter felt ; pain- } \\
\text { less }\end{array}$ & None \\
\hline 5 & $\begin{array}{l}\text { Catheter felt ; pain- } \\
\text { less }\end{array}$ & None \\
\hline 6 & Unknown & None \\
\hline 7 & Nothing felt & None \\
\hline 8 & $\begin{array}{l}\text { Catheter felt ; pain- } \\
\text { less }\end{array}$ & None \\
\hline 9 & $\begin{array}{l}\text { Catheter felt ; pain- } \\
\text { less }\end{array}$ & None \\
\hline 10 & $\begin{array}{l}\text { Catheter felt ; pain- } \\
\text { less }\end{array}$ & $\begin{array}{l}\text { Tingling and itching in } \\
\text { penis }\end{array}$ \\
\hline 11 & $\begin{array}{l}\text { Catheter felt ; pain- } \\
\text { less }\end{array}$ & None \\
\hline 12 & Nothing felt & None \\
\hline 13 & $\begin{array}{l}\text { Slight desire to mic- } \\
\text { turate }\end{array}$ & Slight desire to micturate \\
\hline 14 & $\begin{array}{l}\text { Catheter felt ; pain- } \\
\text { less }\end{array}$ & $\begin{array}{l}\text { Feeling that she is about to } \\
\text { micturate, or that she is } \\
\text { passing urine, or the } \\
\text { normal desire to mic- } \\
\text { turate }\end{array}$ \\
\hline 15 & Nothing felt & None \\
\hline 16 & Nothing felt & $\begin{array}{l}\text { Slight tingling in region of } \\
\text { b.adder }\end{array}$ \\
\hline 17 & $\begin{array}{l}\text { Catheter felt ; pain- } \\
\text { less }\end{array}$ & $\begin{array}{l}\text { Feeling that she is about } \\
\text { to micturate }\end{array}$ \\
\hline 19 & $\begin{array}{l}\text { Tickle in penis ; } \\
\text { pricking at meatus; } \\
\text { desire to micturate }\end{array}$ & $\begin{array}{l}\text { Tickle in penis ; pricking } \\
\text { at meatus; desire to } \\
\text { micturate; pricking } \\
\text { above pubis; feeling } \\
\text { that he is micturating; } \\
\text { feeling that he is stop- } \\
\text { ping himself micturating }\end{array}$ \\
\hline
\end{tabular}


cases which are of particular value in delimiting the tracts are shown in the photographs on Plates I to IV.

In most cases the operation was performed at the fourth, fifth, or sixth thoracic segments. The position and the extent of the incision were purposely varied. It is for this reason that the extent and area of destruction of the cord at the level of the incisions differ greatly from side to side and from case to case.

The degenerated area in the region of the operation is far more extensive than at higher levels ; for the incisions made into the cord may destroy not only long ascending fibres, but also descending and intersegmental fibres. Retrograde degeneration in these descending tracts usually does not extend cranially for more than about a centimetre; and so a true picture of degenerating ascending fibres is obtained cranial to this level.

Photographs of sections from representative cases are shown in Plates I to III. As far as possible the sections have been taken at comparable levels ; in most cases they are from a mid-thoracic segment (usually the site of operation) and from lower and upper cervical segments. The considerable variation in the size of the cords seen in the photographs had been observed also at necropsy.

Figs. 4, 12, 13, and 14 are of sections stained by the Weigert Pal method (degenerating fibres unstained). Fig. $8 \mathrm{c}$ is a Gros' silver preparation showing absence of degenerated axis cylinders. All other figures are of sections stained by the Marchi method; degenerating fibres stained black.

Group 1 (a).-This group consists of cases in which the pathways conducting the sensation of a full bladder and pain from the bladder are bilaterally divided : $1,2,3,4,5,6,7,8,9,10,11,12,13,14$, $15,16,17,18$; Case 19 will be considered in this group.

Cases 1, 2, 3, 4, 5, 6, 7, 8, 9, 10, 11, 12, 13, 14, and 19 were examined histologically. Photographs of representative sections from six of these 15 cases examined histologically are shown in Plates I and II. In 13 of these cases there is no evidence of appreciable degeneration in the posterior columns. It is evident that the afferent pathways must lie within the area of degeneration that is common to all the sections at comparable levels. It therefore lies within the degenerated area in the lateral columns.

The limits of the common area of degeneration at the mid-thoracic level are shown most clearly in Figs. 3, 5, and 6; the anterior limit is shown on the right side of Fig. 5 ; the posterior limit on the right side of Fig. 3, and the left side of
Fig. 6. The common area is shown in Fig. 15; it lies on the surface of the cord, opposite the lateral horn.

Fig. 16 shows the common area of degneration at the upper thoracic level, deduced from an examination of all 15 cases. This area maintains the same position in the cord, as at the mid-thoracic level.

At the low cervical level the common area of degeneration is shown most clearly in Figs. 4, 5, 6, and 7. The anterior limit may be determined from the right side of Figs. 4 and 5, and the posterior limit from the right side of Fig. 4, and the right side of Fig. 6. Fig. 7 suggests that the common degenerated area is a fairly narrow band. This common degenerated area lies on the surface of the cord approximately opposite the posterior angle of the anterior horn (Fig. 17). At the high cervical level the area of degeneration is fairly constant throughout the cases. It is in the form of a band extending from the posterior horn to the anterior lateral fissure approximately. Figs. 4 and 6 show that this band is divided into two by an area of intact fibres, lying a little posterior to the level of the lateral dorsal process of the anterior horn.

From the 15 cases so far discussed it would not be possible to determine within which part of the degenerated area the afferent pathway from the bladder runs. Cases 21, 22, and 28 allow more accurate localization to be made. In none of these cases was there any disturbance of bladder sensation. All three cases show degeneration in the most posterior zone of the lateral columns on the surface of the cord; this degeneration is particularly marked in Figs. 10 and 11. This degenerated area corresponds to the more posterior zone of degeneration described in Figs. 4 and 6 . Therefore the afferent fibres under discussion cannot lie in this area, but must run in the more anterior zone of degeneration, approximately opposite the lateral dorsal process of the anterior horn (Fig. 18).

Figs. 15 to 18 show the smallest common area of degeneration at different levels of the cord in the cases with loss of the sensation underlying the desire to micturate. This area keeps in approximately the same position on the surface of the cord throughout the region studied (cervical and upper thoracic); in the thoracic region it is opposite the lateral horn, and in the cervical region opposite the lateral dorsal process of the anterior horn.

Case 19 (Fig. 8) is particularly interesting, as pain from over-distension of the bladder was felt, and, occasionally, the patient stated that he had a desire to micturate. The section of the mid-cervical region shows the area of operation on the left side of the cord. It is possible that the area of degenera- 


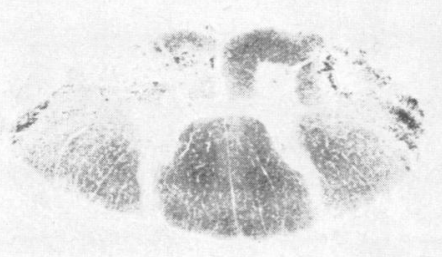

a

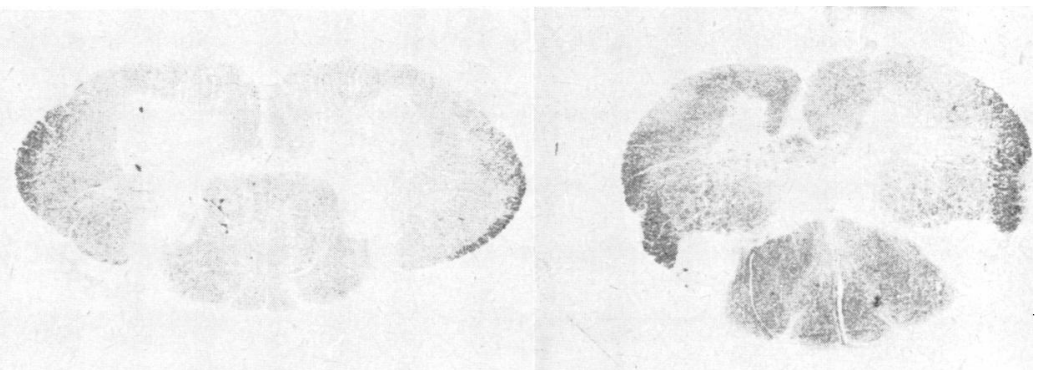

b

Fig. 3. (Case 1). a : Th.4. b : C7. c: C1.

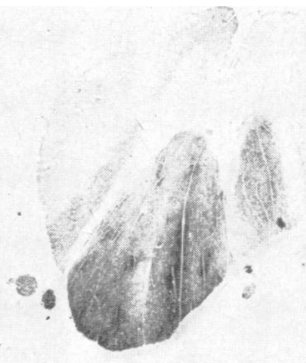

a

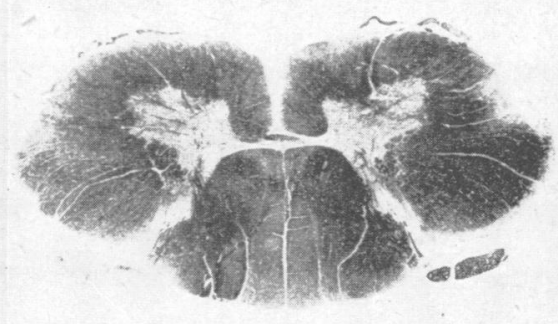

b

Fig. 4. (Case 5). a : Th.7. b: C7. c: C2.

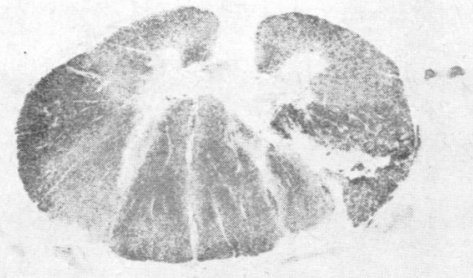

a

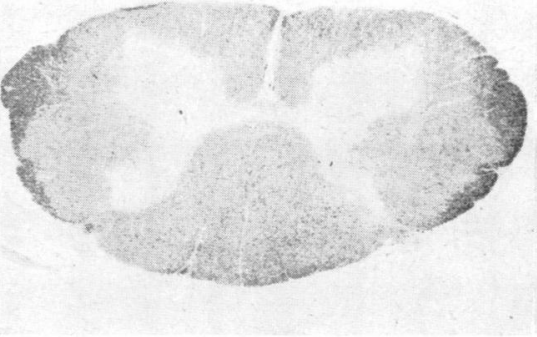

b

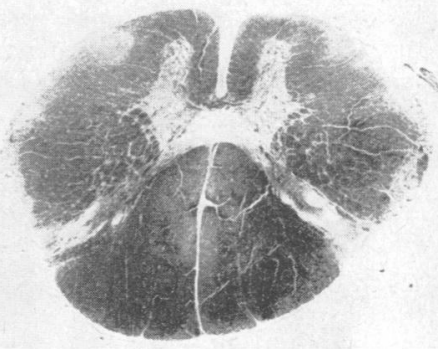

c

Fig. 5. (Case 6), a: Th.5 and 6. b: C7. c: C1.

All figures are photographs of transverse sections of spinal cords $(\times 4 \cdot 25)$ stained to show areas of fibre degeneration. In Figs. 4, 12, 13, and 14 the sections are stained by the Weigert Pal method.

Figs. 3-8 are from cases with bilateral lesions of the antero-lateral columns involving centripetal pathways from bladder and some centripetal pathways from urethra. In Figs. 3-11, " $a$ " show's the region of operation.

Plate I

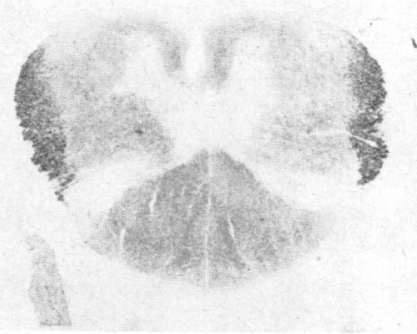

c

\section{ญำ임 \\ 需 \\ 웅

꿍요 


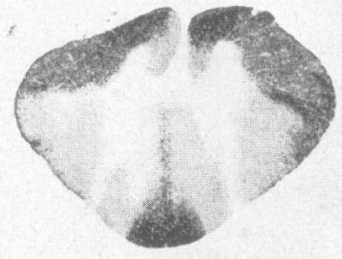

a

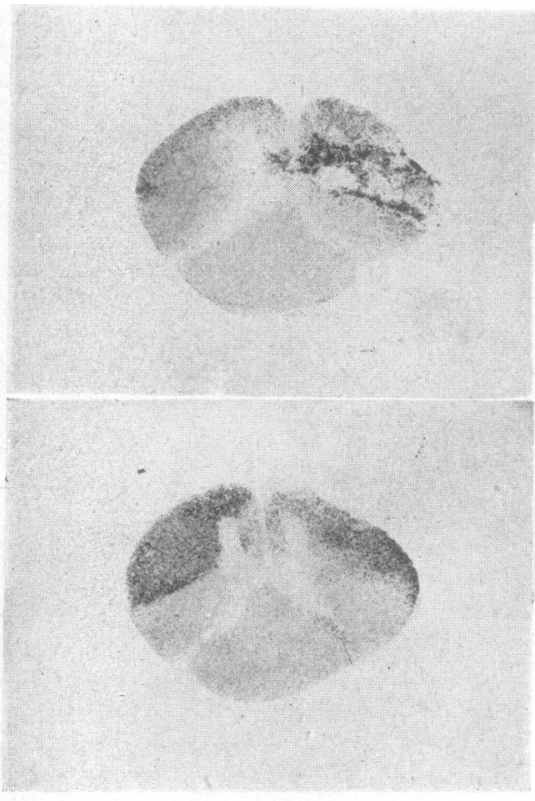

Fig. 6. (Case 10), a: Th.5. b: C7. c: C1.
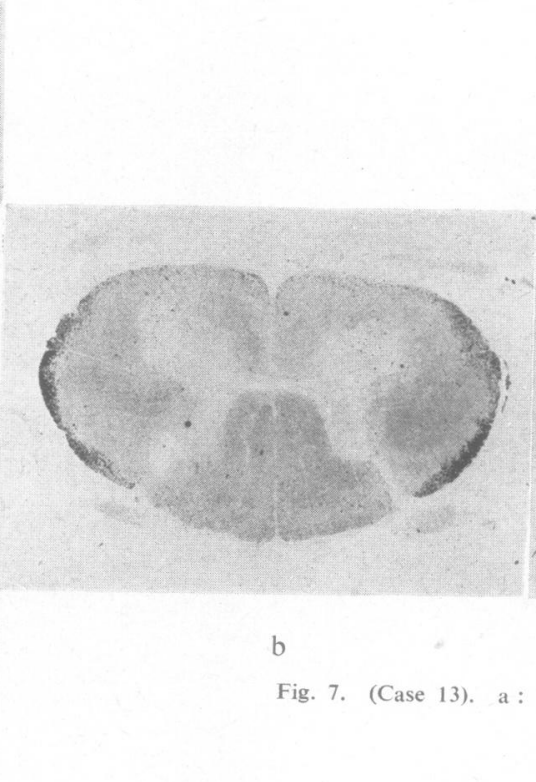

b

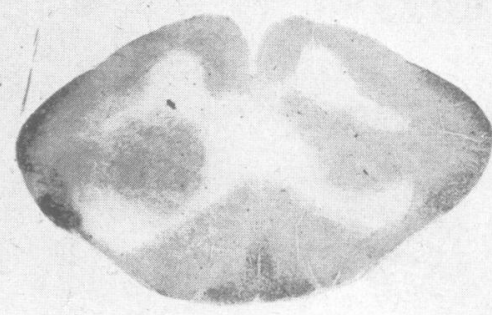

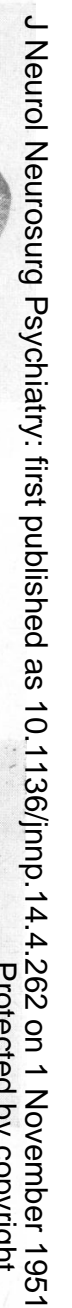

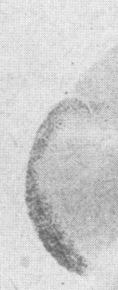

Fig. 7. (Case 13), a: Th.9. b: C7. c : Cl.
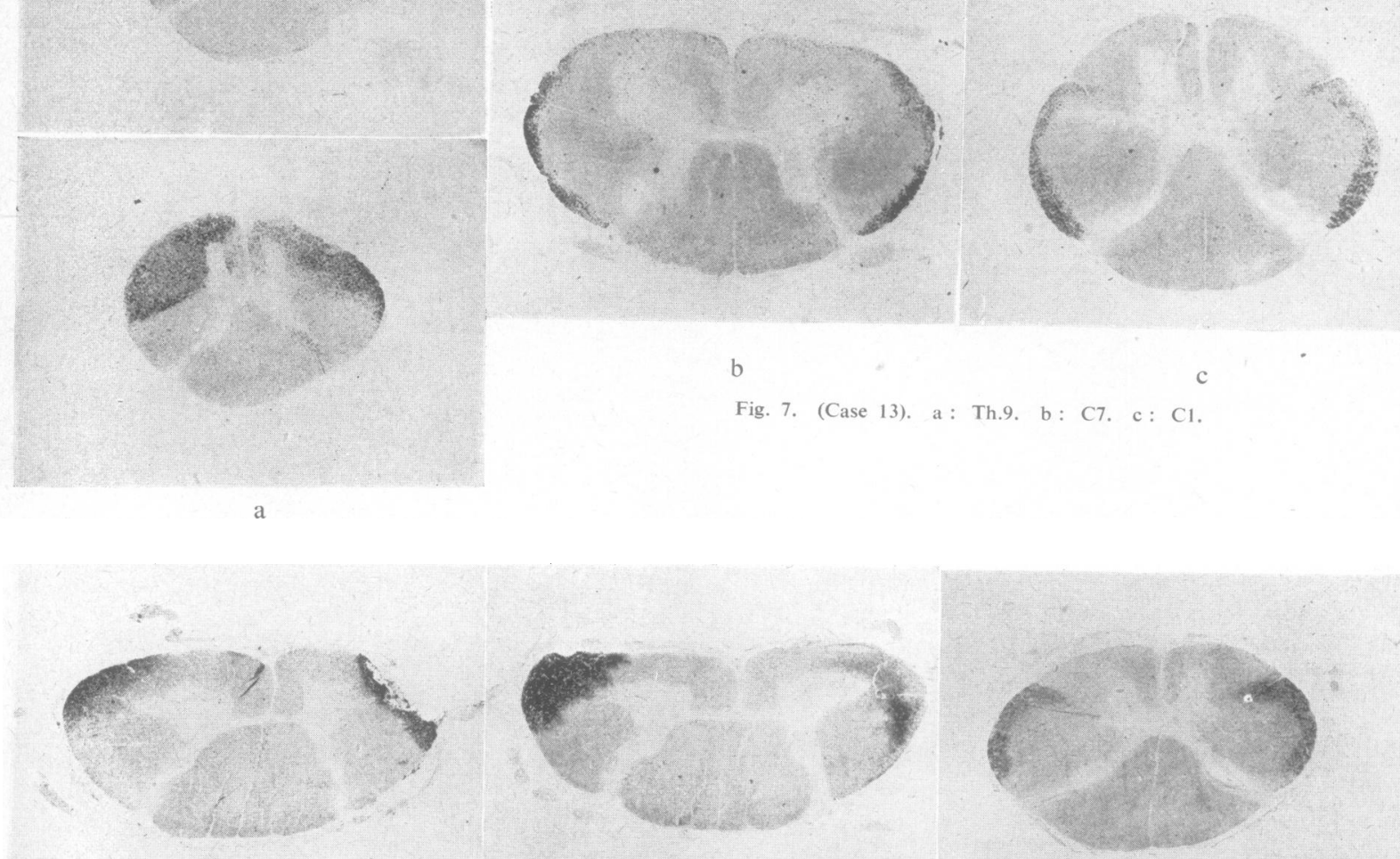

a

Fig. $8 \mathrm{c}$ is a Gros silver preparation. In all other Figures the sections are stained $b y$ the Marchi method.

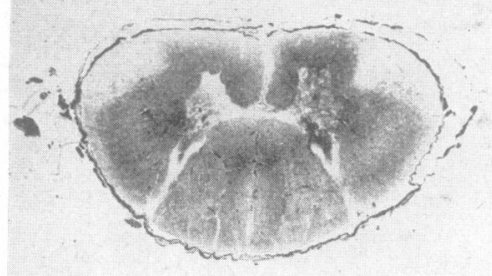

Fig. 8. (Case 19), a : C5 and 6. b: C4. c: $\mathrm{Cl}$.

Plate II 
Figs. 9, 10, and 11 are from cases with lesions of the antero-lateral columns, with partial or no involvement of the centripetal pathways.

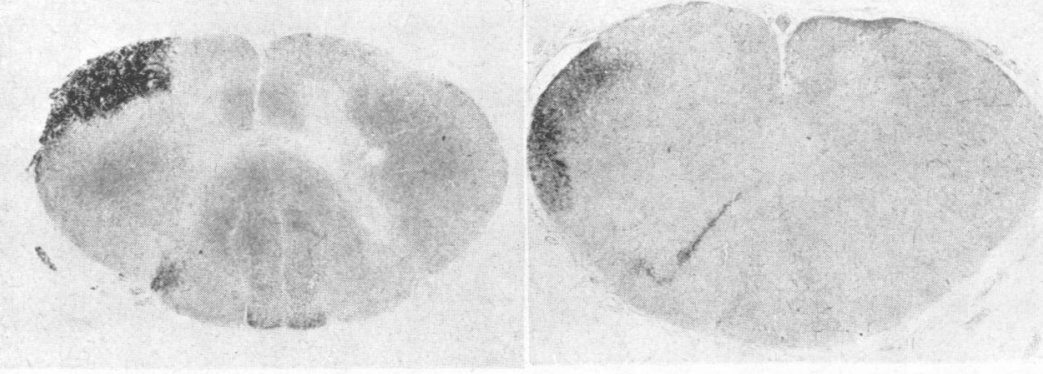

a

b

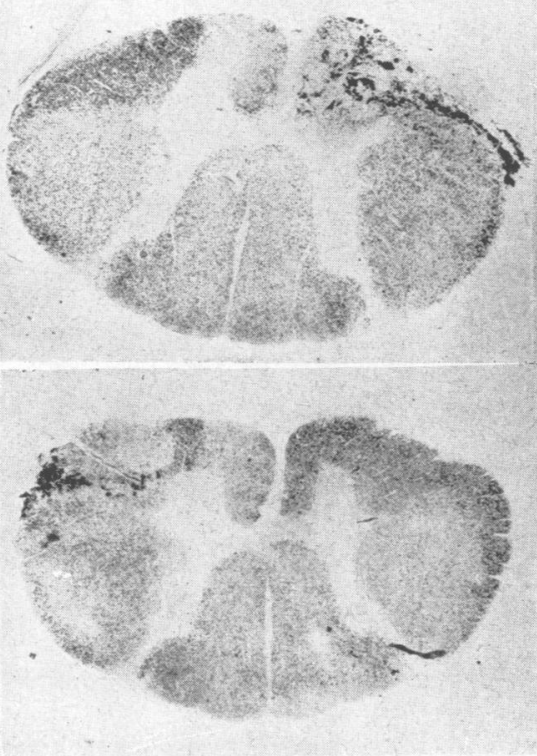

Fig. 9. (Case 20). a : C3. b: C1.

a

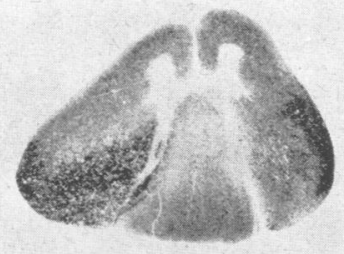

b

Fig. 10. (Case 21), a : Th.1. b: C7. c: C1.
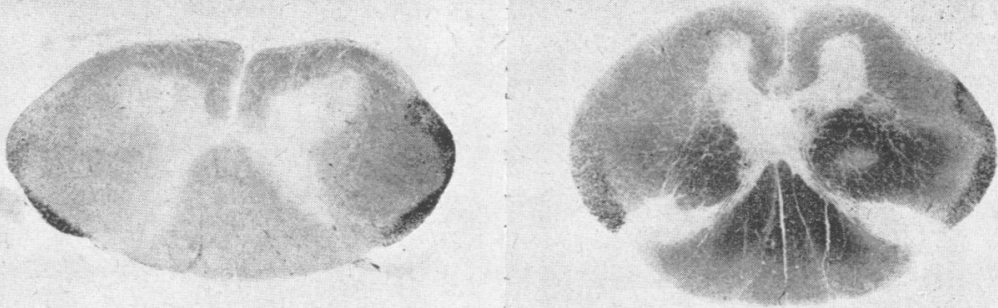

b

c

Fig. 11. (Case 22). a: Th.5. b: C7. c : C1.

9. 


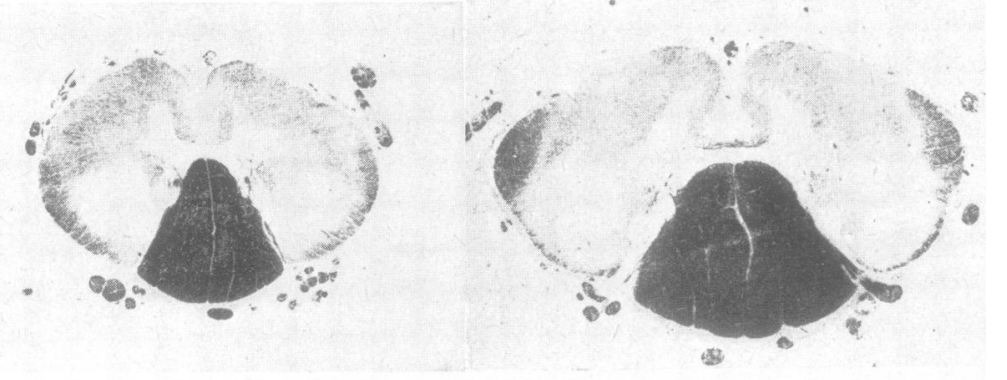

a

Fig. 12. (Case 28). a : Th.5. b: C3.
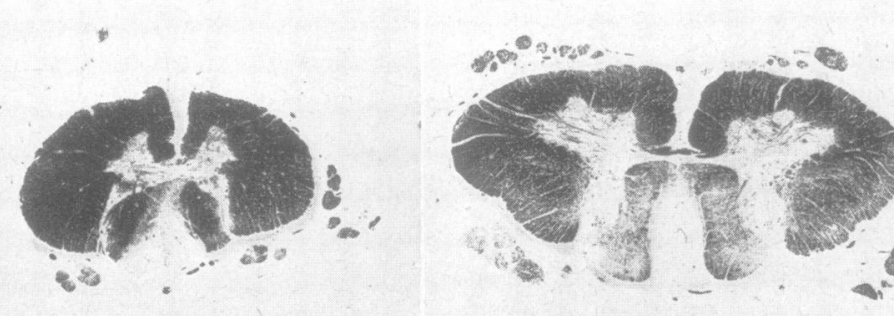

b

Fig. 13. (Case 29). a: Th.2. b: C7. c: C4.
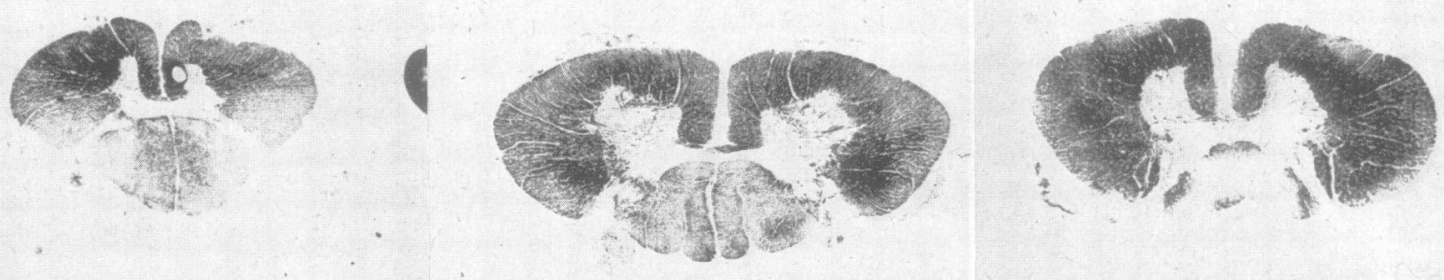

b

Fig. 14. (Case 30). a : Th.4. b : C7. c : C4.

Figs. 12, 13, and 14 are from cases with lesions not involving the centripetal pathways. 
tion on the left side does not extend as far posteriorly as the total extent of the area within which the afferent pathway lies. It is possible that a few fibres in this area were not divided. This suggestion is supported by the Gros' silver preparation of the spinal cord just rostral to the operation area. Sections prepared by this method to demonstrate axis cylinders show more precisely the limits of destruction of fibres. From these silver sections it can be seen that the area of destruction does not extend as far posteriorly as the limit of the common pathway.

Group 1(b).- - This group consists of cases in which the pathways conducting the sensation of a full bladder and pain from the bladder are bilaterally intact : 28, 29, and 30 . All these cases were examined histologically.

Photographs of representative sections are shown on Plate IV. This group consists of the cases that form the control. They provide further evidence of the region where these afferent fibres do not run. In Fig. 12 there is almost complete degeneration of the posterior half of the lateral columns in the high cervical level. There is no degeneration in this case in the area of the lateral columns, or on the surface of the cord opposite the lateral dorsal process of the anterior horn, where it has been shown that the afferent pathway lies. In Fig. 13 there is almost complete degeneration in the funiculi graciles, a very few anterior fibres being spared. In Fig. 14 at the high cervical level the funiculi graciles are degenerated, except for a few posterior fibres ; the mid-zone of the funiculi cuneati are also degenerated. No bladder dysfunction of any kind occurred in these cases, confirming that no afferent pathway from the bladder runs in the posterior columns.

Group 1(c).- - This group consists of cases in which the pathways conducting the sensation of a full bladder and pain from the bladder are unilaterally divided : 20, 21, 22, 23, 24, 25, and 26.

Cases 20, 21, and 22 were examined histologically. Photographs of representative sections are shown on Plate III. In Case 20 (Fig. 9), the operation was unilateral; although the degeneration includes that common area demonstrated in Group 1(a), the sensations of needing to micturate and of pain were normal. In Case 21 (Fig. 10), the area destroyed at operation is very anterior ; in Case 22 (Fig. 11) it is very posterior. In these two cases the pathway under discussion may have been sectioned on one side, but on the other side it was definitely intact. In Fig. 10 the degeneration on the left side does not involve the region under discussion; in Fig. 11 the degenerated area on the right side might include this afferent pathway, but on the left side the pathway is definitely not involved. Thus, as far as this afferent pathway is concerned, these two cases may be treated as unilateral cases. They confirm the findings from Case 20, which shows that when the afferent pathway is divided unilaterally the subject still remains aware of the state of distension of his bladder and has the sensation of needing to micturate and the pain of over-distension.

Group 2(a).-This group consists of cases in which the pathways conducting the sensations of pain and temperature from the urethra are bilaterally divided : $1,2,3,4,5,6,7,9,10,11,12,13,14,15,16$, and 19 . Cases 1, 2, 3, 4, 5, 6, 7, 9, 10, 11, 12, 13, 14, and 19 were examined histologically. Photographs of sections from representative cases are shown in Plates I and II. These are the same cases as have been discussed in Group 1(a), without Case 8. The smallest common area of degeneration was determined in the section dealing with Group 1(a). It is thus evident that the pathway conducting the sensations of pain and temperature from the bladder and the urethra lies within the same area of degeneration as the pathways conducting the sensation of a full bladder (Figs. 15 to 18).

Group 2(b).--This group consists of cases in which the pathways conducting the sensations of pain and temperature from the urethra are unilaterally divided : 8, 17, 20, 21, 23, and 24 .

Cases 8,20 , and 21 were examined histologically. Photographs of representative sections of Cases 20 and 21 are shown in Plate III. These cases have already been considered under Group 1(c). It is seen that the pathway conducting impulses subserving pain and temperature from the urethra runs in the same region as that conducting impulses subserving the sensation of a full bladder and pain from the bladder.

\section{Discussion}

Seventeen patients with bilateral lesions of the spino-thalamic tract had lost the normal sensation underlying the desire to micturate, and the sensations of pain and temperature from the bladder and urethra. The pathway in the cord conducting the impulses giving rise to these sensations lies on the surface of the cord, approximately opposite the dorsal lateral process of the anterior horn. It lies in the same region of the cord as that conveying impulses interpreted as pain, warmth, and cold from the sacral segments of the body.

A control group of cases without lesions of the spino-thalamic tract has been given; these cases had degeneration of spinal tracts, with normal bladder and urethral sensation. A case of subacute 
combined degeneration of the cord (Case 29), and a case of Friedreich's ataxia (Case 30) show degeneration of afferent pathways with no disturbance of the sensations that concern us here. The inclusion of a case of amyotrophic lateral sclerosis (Case 28), a motor and not a sensory disorder, is justified on similar grounds: the lesions in the spinal cord serve to show where the tracts do not run. These few cases were included because clear histological specimens were available. Many other cases have been seen, with similar lesions not involving this pathway. Such patients had had no abnormality or lack of sensation from the bladder.

Thus the evidence presented shows that the centripetal tract in man lies in a region of the cord similar to where it lies in the cat. In man its position is somewhat more lateral, for it lies within the most lateral region of the cord, about midway between the anterior and posterior horns (Figs. 15 to 18). It should be possible to narrow down the region occupied by this tract still further, as more evidence is collected.

The only other evidence known to us is that of the case reported by McMichael. In his specimen the tract could not be identified in the cervical region, and was not clear in the thoracic region. In the lumbar region-the region that could not be investigated in our series of cordotomies-" Weigert Pal sections showed a bilaterally symmetrical zone of degeneration immediately anterior to the posterior cornua lying superficially in the lateral columns". This region is similar to that in which the pathway has been located in the present investigation in the thoracic and cervical regions; but in the cases presented here, at the higher levels, it is more anterior and more lateral ; for there is an area free from degeneration immediately anterior to the posterior cornua. McMichael's case is particularly im-

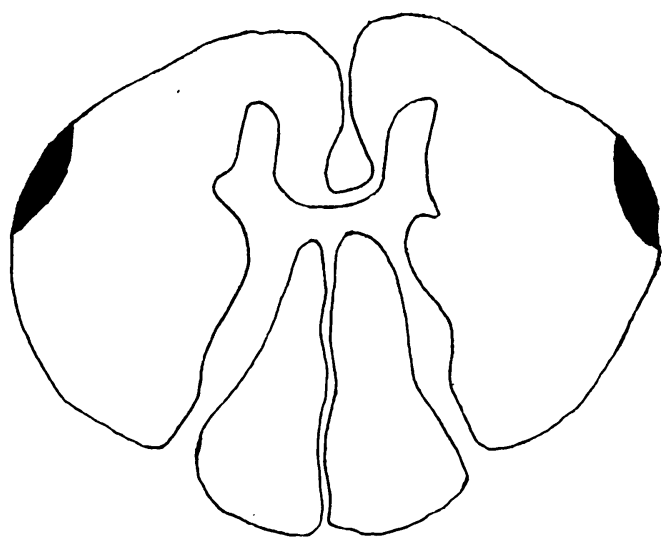

Fig. 15.-Mid-thoracic region.

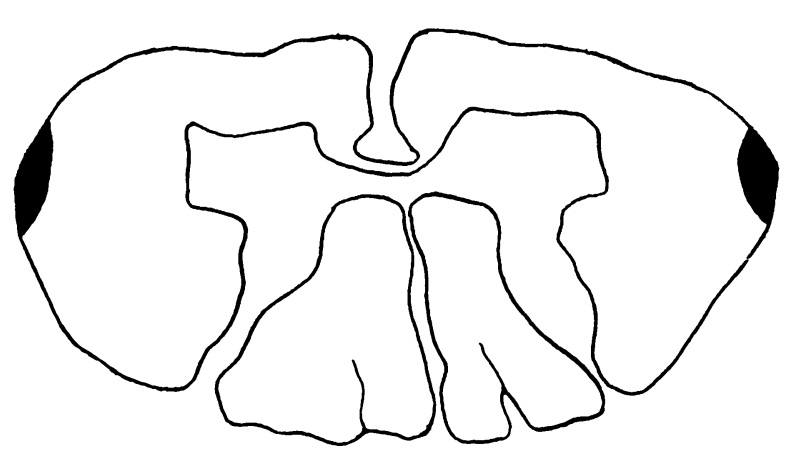

Fig. 17.-Lower cervical region.

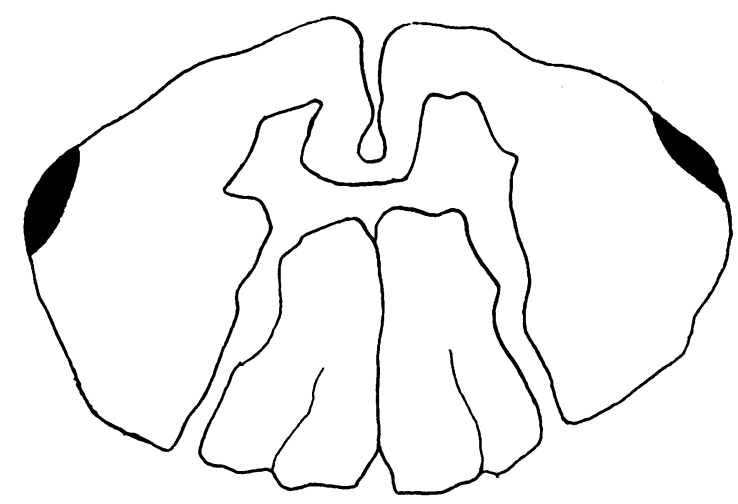

Fig. 16.-Upper thoracic region.

Figs. 15-18.-Drawings of transverse sections of the spinal cord. The area containing the centripetal pathway from the bladder and the spincthalamic pathway from the urethra is shaded. 
portant, as the patient had no somatic sensory loss, for it shows that the part of the lateral column conveying the centripetal path from the bladder can be divided, without there being a simultaneous division of the somatic centripetal pathway in the spino-thalamic tract.

Most patients having a unilateral lesion of the spino-thalamic tract notice no change in bladder function, and retain the normal sensation of a full bladder and desire to micturate. In all, 12 cases of unilateral cordotomy have been studied; seven are not included in this series, as they added nothing to those described here. In five of them (Cases 20, $23,25,26$, and 27) for some time the sensation giving rise to the desire to micturate was experienced unilaterally; in the others no abnormality was noted. Direct questioning on the laterality of this sensation was not used, because the possibility of the sensation being unilateral had not been thought of until Case 23 was seen. Before this investigation was begun, three cases of thrombosis of the posterior inferior cerebellar artery had been studied. These patients were not questioned about the desire to micturate; none of them spontaneously made any comments on it, or on urethral sensation. Towards the end of this investigation a further case (Case 24) was encountered. This patient in reply to questioning stated that he had the normal sensations underlying the desire to micturate, and that he felt his urine passing in the normal manner. He was then investigated in accordance with the scheme employed for the cases of this series. The patient then noticed that when he was catheterized, he felt the passage of the catheter only on the left, which was the side of the body having normal sensation. As his bladder was filled, he was then surprised to find that he experienced the desire to micturate only on the left. From the experience of this case, one is inclined to wonder whether the other cases of posterior inferior cerebellar artery thrombosis might not have had this sensation unilaterally had their attention been directed to their sensations ; during cystometrography there is ample time for observing sensations while the bladder is being slowly filled. Two of the patients with unilateral cordotomy (Cases 26 and 27) experienced this sensation unilaterally only when the desire to micturate became urgent enough to force itself on their attention.

To account for the discrepancy in the evidence of these six patients, who felt the sensation underlying the desire to micturate unilaterally, and that of other patients with a unilateral lesion feeling the sensation centrally and not unilaterally, one may consider two possibilities. It could be that a vague and poorly localized sensation, such as that underlying the desire to micturate, is felt centrally and bilaterally, whatever the anatomical pathway subserving it ; for it could be that for a sensation that has for years been central and bilateral a pattern of perception has become so fixed that it over-rides the perception of an innovation in the sensation. Or it might be that, in some cases, this pathway from one side of the bladder runs up both sides of the cord, while in others it runs up the opposite side of the cord only.

From unilateral cases, it was learnt that the pathway conducting impulses subserving the sensation of pain from the lower ends of the ureters, from the walls of the bladder, from the urethra, and thermal sensation from the urethra, also runs in the spino-thalamic tract, and in the same region as that conducting the sensation underlying the desire to micturate.

Patients in whom the pathway in the spinothalamic tract has been divided, may retain the sensation that micturition is impending, that it is progressing, and that it has ceased. These sensations are doubtless due to impulses arising in the urethral mucosa and in the sphincter urethrae membranaceae and the large group of perineal muscles that act synergically with the sphincter. Such impulses doubtless travel via the pcsterior columns.

It remains to answer the question : why do not all patients, after bilateral spino-thalamic cordotomy, have urethral sensation, giving them knowledge that micturition is starting, progressing, and stopping.

Immediately after division of both spino-thalamic tracts patients may have no sensation from bladder or urethra. This occurs more commonly in women than in men, and is likely to be due to the fact that women have shorter urethras than men, with less length of mucosa. This condition may last a month or more, and patients who die in this period have therefore regained no urethral sensation. Those living past this stage tend to feel more. The removal, by division of the spino-thalamic tracts, of all sensation from the bladder, and the greater part of sensation from the urethra-for the sensations of pain and warmth and cold are the predominant urethral sensations-demands from the patients a re-orientation to the sensations perceived. Similarly in the sphere of somatic sensation, many patients lose the ability to tell sharp from blunt ; but others do not. Also, the feeling of wetness is difficult to perceive after spino-thalamic division, doubtless due to the removal of thermal sense; but some patients learn to know when something is wet, while others do not-both having the same sensory apparatus. After cordotomy a new sensory pattern from the urethra is presented to consciousness; 
not all patients become equally adept in interpreting it. Its interpretation depends on the ability to carry out minute observations on one's own sensations. Further, the patients' ability to explain what they feel enters into the question of what sensation is regained; probably patients often experience sensations of greater complication than they are able to describe.

As has been noted in the histories of these patients, there is a vagüe sensation related to distension or rise in pressure in the bladder, which we have called, non-committally, a substitute sensation. We do not propose discussing this sensation further here ; work is continuing on the origin of the sensation, and the pathway by which the impulses subserving it reach the cerebrum. It is sufficient to point out that wherever these impulses arise, they do not travel in the spino-thalamic tract.

It remains to discuss Case 19, the patient who lost the normal desire to micturate, and who was found, within 24 hours of the operation that cut off the sensation of pain from the rest of his body below the fourth thoracic segment, to be suffering from great pain due to distension of the bladder. Although there was good recovery of urethral sensation, urethral pain was not regained ; and so the pain of distension of the bladder cannot be attributed to urethral sensation. As has been mentioned when considering the histological evidence, it is probable that a few fibres, at least on one side, lying posteriorly, escaped cutting at operation. One is unwilling to draw too definite a conclusion from one case, with regard to a possible separation of pathways subserving pain and pathways subserving the desire to micturate. As further evidence is collected, it may become possible to draw further conclusions about the detailed topography of these tracts.

Before finishing this discussion on pathways of conduction of impulses that are interpreted as sensation, it is of importance to remember that the sensation of having a full bladder that needs to be emptied, can, like all sensations, be evoked by psychic factors. This was shown clearly by one of our patients, the subject of Case 9, who, although she had no sensation from her distended bladder, experienced the sensation of needing to micturate, whenever she saw or heard the bed-pans being taken round the ward.

\section{Summary}

The location within the spinal cord of man of the following afferent pathways has been determined : (a) that subserving the sensation that the bladder is full, giving rise to the desire to micturate ; $(b)$ that subserving the sensations of pain from the bladder, urethra, and lower end of the ureter, and sensation of temperature from the urethra; (c) that subserving touch and pressure or tension from the urethra. The pathways considered under the headings $(a)$ and $(b)$ lie within the spino-thalamic tract on the surface of the cord opposite the dorsal lateral process of the anterior horn. The pathways considered under $(c)$ lie within the posterior columns.

We wish to express our gratitude to Mr. Wylie McKissock for his whole-hearted cooperation; he operated on the majority of these patients, and, while relieving their pain, maintained a scientific attitude, which greatly aided the investigation, of which this paper is the first report.

We are grateful to the many surgeons and pathologists who have helped us in this work; and particularly to Mr. O'Connell, Professor Norman Dott, Mr. Dickson Wright, Mr. Norman Matheson, Mr. E. A. Turner, Mr. Malcolm Donaldson, Dr. J. G. Greenfield, Dr. W. Blackwood, Dr. W. G. C. Mair, and Professor W. D. Newcomb. We wish to thank Mr. P. B. Ryan for carrying out a number of the cystoscopies. We are also grateful to Dr. E. A. Carmichael, who helped us by providing the facilities for this investigation and by his interest and criticism. Our thanks are due to the Medical Committee of the National Hospital for Nervous Diseases for allowing us to use their case records.

We wish also to thank Mr. F. J. F. Barrington and the Editor of Brain for permission to reproduce Fig. 1.

To our technical assistants, Mrs. J. A. Mills and Miss Kathleen Harrison, we wish to express our gratitude for their careful and painstaking work; and we also wish to thank Mr. J. A. Mills for giving us the benefit of his experience of histological techniques and photography.

Patients 6, 11, 13, 19, 24, 25, and 27 were in the National Hospital ; their case numbers were respectively 18299 , $23011,16162,12166,30790,26668$, and 16148.

\section{REFERENCES}

Barrington, F. J. F. (1925). Quart. J. exp. Physiol., 15, 81. (1933). Brain, 56, 126.

Bing, R., and Haymaker, W. (1939). "Textbook of Nervous Disease." St. Louis.

Frankl-Hochwart, L. von, and Zuckerkandl, O. (1906). Die nervösen Erkrankungen der Harnblase, 2nd ed. Vienna.

Guttmann, L., and Whitteridge, D. (1947). Brain, 70, 361. McMichael, J. (1945). Ibid., 68, 162.

Müller, L. R. (1919). Dtsch. Arch. klin. Med., 128, 81. Smith, M. C. (1951). Journal of Neurology, Neurosurgery, and Psychiatry, 14, 222. 